$3 \%$

\title{
Caso
}

Empresarial

10

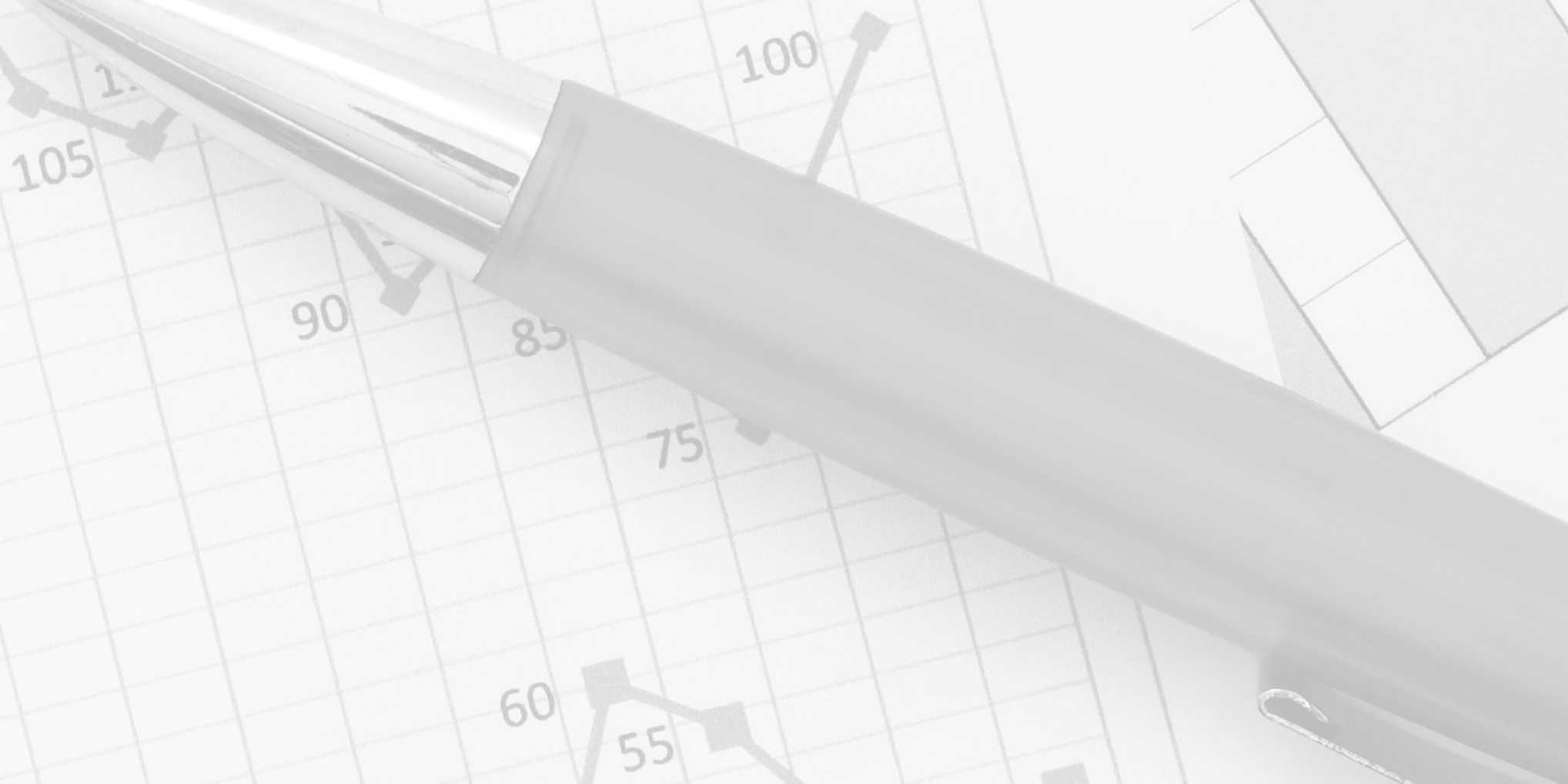





\section{Diagnóstico para la implementación de las herramientas Lean Manufacturing, desde la estrategia de operaciones en algunas empresas del sector textil confección de Colombia: reporte de caso}

DOI: https://doi.org/10.21158/01208160.n85.2018.2058

Fecha de recepción: 05 de febrero de 2018

Fecha de aprobación: 16 de mayo de 2018
Henry Helí González Gaitán ${ }^{1}$ Corporación Universitaria Minuto de Dios - UNIMINUTO

henry.gonzalez@uniminuto.edu

Natalia Marulanda Grisales ${ }^{2}$ Corporación Universitaria Minuto de Dios - UNIMINUTO natalia.marulanda@uniminuto.edu

Francisco Javier Echeverry Correa ${ }^{3}$ Corporación Universitaria Minuto de Dios - UNIMINUTO javier.echeverry@uniminuto.edu

Cómo citar este artículo / To reference this article / Comment citer cet article / Para citar este artigo:

González Gaitán, H. H.; Marulanda Grisales, N. y Echeverry Correa, F. J. (2018). Diagnóstico para la implementación de las herramientas Lean Manufacturing, desde la estrategia de operaciones en algunas empresas del sector textil confección de Colombia: reporte de caso. Revista EAN, 85,199-218. DOI: https://doi.org/10.21158/01208160.n85.2018.2058

\section{Resumen}

La filosofía de Lean Manufacturing hace parte de las metodologías de mejoramiento continuo, que facilitan la gestión sistémica de las organizaciones. Teniendo esto en cuenta, se formula como propósito de este artículo presentar el diagnóstico realizado en torno a la implementación de las herramientas Lean Manufacturing desde la estrategia de operaciones, dando a conocer de esta manera los factores relevantes que intervienen en la implementación de las mismas, todo lo anterior a partir de los hallazgos de la investigación realizada en algunas empresas del sector textil confecciones de la subregión del Valle de Aburrá, del departamento de Antioquia, en Colombia. Esta investigación tuvo un carácter mixto, enfocado en un estudio de caso, para el que se utilizó una muestra por conveniencia y un cuestionario de preguntas estructuradas, el cual permitió identificar las variables que favorecen la mejorar la productividad y rentabilidad de las empresas en el largo plazo.

\section{Palabras clave}

Producción limpia, administración de la producción, manufacturas - metodología, gestión industrial, industria textil - Antioquia - Colombia.

\footnotetext{
${ }^{1}$ Ingeniero industrial, Universidad de Antioquia. Especialista en Logística Integral, Universidad de Antioquia. Especialista en Gestión Energética Industrial, Instituto Tecnológico Metropolitano ITM. Magister en Educación Docencia, Universidad de Manizales. ORCID: https://orcid.org/0000-0002-4937-907X

2 Ingeniera Industrial, Universidad Nacional de Colombia. Magister en Ingeniería Industrial, Universidad Nacional de Colombia. Estudiante de Doctorado en Ingeniería - Industria y Organizaciones, Universidad Nacional de Colombia, Sede Medellín. ORCID: https://orcid.org/0000-0002-9229-6771

${ }^{3}$ Administrador de Negocios Universidad, de EAFIT. Especialista en Mercadeo, de EAFIT. Magister en Educación, Universidad de Manizales. ORCID: https://orcid.org/0000-0002-2482-734X
} 


\title{
Diagnosis in the implementation of Lean manufacturing tools based on the operation strategy in some companies from the textile sector in Colombia: a case report
}

\begin{abstract}
The philosophy of Lean Manufacturing is part of the continuing improvement methodologies which facilitate organizational systemic management. Based on this, this article aims at showing the diagnosis made in the implementation of Lean Manufacturing tools from an operation strategy perspective, which describes the main factors that intervene in their implementation process. This is possible thanks to the research findings in some companies from the textile sector in the area of Valle de Aburra, in the department of Antioquia, Colombia. This research study has a mixed character based on a case study in which a convenience sample and a structured questionnaire are used to identify the variables which favor company productivity and profitability at a long term.
\end{abstract}

Key words. CLean production, production management, manufacturing- methodology, industrial management, textile industry-Valle de Aburra, Antioquia, Colombia.

\section{Diagnostic de la mise en place d'outils de fabrication eco-responsables dans la stratégie opérative des entreprises du secteur textile colombien: étude de cas}

Résumé. La philosophie d'une fabrication eco-responsable fait partie des méthodologies d'amélioration continue facilitant la gestion systémique des organisations. Cet article présente un diagnostic et les facteurs pertinents pour la mise en oeuvre d'une stratégie opérationnelle utilisant des outils de fabrication eco-responsables. Cette étude s'appuie sur les conclusions des recherches effectuées dans certaines entreprises textiles colombiennes de la région de la vallée d'Aburrá - Antioquia. Cette investigation à caractère mixte se base sur l'echantillon d'une étude de cas et un questionnaire structuré permettant d'identifier les variables favorables à l'amélioration de la productivité et de la rentabilité des entreprises sur le long terme.

Mots clefs. EProduction propre, gestion de la production, manufactures - méthodologie, gestion industrielle, industrie textile - Antioquia-Colombie.

\section{Diagnóstico para a implementação das ferramentas Lean Manufacturing, a partir da estratégia de operações em algumas empresas do setor têxtil confecção da Colômbia: relatório de caso}

Resumo. A filosofia de Lean Manufacturing faz parte das metodologias de melhoria contínua, que facilitam o gerenciamento sistémico das organizações. Considerando isto, formula-se como propósito deste artigo apresentar o diagnóstico realizado em torno da implementação das ferramentas Lean Manufacturing a partir da estratégia de operações, dando a conhecer desta maneira os fatores relevantes que intervêm na implementação das mesmas, tudo isto a partir das descobertas da pesquisa realizada em algumas empresas do setor têxtil confecções da sub-região do Vale de Aburrá, do departamento de Antioquia, na Colômbia. Esta pesquisa teve um caráter misto, focado em um estudo de caso, para o que se utilizou uma mostra por conveniência e um questionário de perguntas estruturadas, o qual permitiu identificar as variáveis que favorecem o melhoramento da produtividade e da rentabilidade das empresas a longo prazo.

Palavras-chave. Produção limpa; administração da produção, manufaturas - metodologia, gerenciamento industrial, indústria têxtil -- Antioquia - Colômbia. 


\section{Introducción}

$\mathrm{n}$ el desarrollo del presente artículo,
en primer lugar se abordan conceptos fundamentales en la teoría de Lean Manufacturing (LM), y su papel dentro de las diferentes estrategias de mejoramiento continuo que se aplican en el día a día a través de la implementación de la filosofía LM en todas las áreas de las empresas; luego del análisis de este concepto, se consideran otras teorías fundamentales en la implementación de LM tales como LA estrategia de operaciones, las herramientas de manufactura esbelta como Kanban, 5S, desarrollo de proveedores, definición del direccionamiento de la empresa, diagnóstico, objetivos de largo plazo, estrategia de operaciones de calidad y costos, entre otros. Se termina el artículo con la exposición de la metodología desarrollada en el estudio y el consecuente análisis de resultados, que derivan en las propuestas y las conclusiones del estudio de caso.

La CEPAL expresa que "para América Latina, su producción está mayormente vinculada con el mercado interno por lo que una parte significativa de la población y de la economía de la región depende de su actividad» (2010, p. 13).

En correspondencia con lo anterior, la manufactura esbelta se puede considerar como una estrategia para mitigar algunas de las ineficiencias en el uso de recursos, ya que al aplicar las técnicas y herramientas propuestas por Lean se logra aumentar la rentabilidad de los activos hasta en un $100 \%$, reducir los tiempos de entrega en un $99 \%$, aumentar la disponibilidad de las máquinas un $95 \%$, reducir los inventarios en un $75 \%$, entre otros beneficios (Vienazindiene y Ciarniene, 2013).

Por otro lado, la aplicación de técnicas, herramientas o filosofías es escaso en pequeñas y medianas empresas, por lo que resulta importante identificar los elementos que contribuyen a que las pymes ${ }^{4}$ dedicadas a la confección en la ciudad de Medellín, puedan presentar avances importantes en su implementación, ya que en la actualidad solo las grandes empresas obtienen beneficios al ejecutarlas (Pérez, Patiño y Úsuga, 2010).

El artículo busca además mostrar de manera más concreta, en especial dentro del área de logística, los hallazgos surgidos del cumplimiento de los objetivos específicos del estudio de caso en mención, desde los cuales se lograron establecer, mediante el cruce de variables, los factores de mayor impacto en el desarrollo de las empresas; fruto del análisis de la correlación entre estos factores, se pudo igualmente identificar los factores cualitativos y cuantitativos intervinientes, mostrando aquellos que tienen mayor impacto para mejorar la productividad y rentabilidad de dichas compañías: Para tal efecto, se llevó a cabo la investigación de carácter mixto, bajo el enfoque de un estudio de caso, usando una muestra por conveniencia, diligenciando una encuesta estructurada, y la aplicación del software Statgraphics Centurion; finalmente,

\footnotetext{
${ }^{4}$ Sigla definida en la Ley 590 del año 2000 y que concretamente hace relación a la micro, pequeña y mediana empresa en Colombia
} 
dentro de los resultados parciales derivados del estudio, en este artículo se comparten dos propuestas de orden administrativo, realizadas por los autores, basadas en la estrategia de operaciones, calidad y costos, las cuales permiten la optimización en la implementación de herramientas LM, en beneficio de la eficiencia y eficacia de los procesos productivos de las empresas.

\section{Filosofía Lean, la organización y gestión}

L a estrategia de operaciones se centra en la toma de decisiones para facilitar los procesos de transformación que convierten los insumos en productos o servicios deseados, con respecto a los procesos, la calidad, capacidad e inventario. Esta estrategia se encuentra soportada en gran medida por las actividades de logística, mercadeo y finanzas de cada empresa.

De acuerdo con Alukal y Manos (2006, p.1), Lean es «una filosofía de manufactura o administración que disminuye los tiempos de espera entre la orden de un cliente y el envío de las partes o servicios ordenados, llevando a las compañías a ser más competitivas y ágiles». Por su parte, Madariaga (2013) y Rajadell y Sánchez (2010), sugieren que el Lean Manufacturing es un modelo de organización y gestión del sistema de fabricación -personas, materiales, máquinas y métodos- que por medio de la mejora continua persigue incrementar la calidad, el servicio y la eficiencia mediante la identificación y eliminación del desperdicio o despilfarro. Con Arrieta et al. (2010, p.144) se reconfirma que «el objetivo del Lean Manufacturing es identificar y eliminar los posibles desperdicios en el momento en que se identifiquen, para lograr la mejora de la productividad».

En este sentido, es importante hacer estudios que permitan comprender cuáles son las variables de algunas empresas colombianas ubicadas en el Valle de Aburrá que al implementar la filosofía Lean, desde la estrategia operacional, de calidad y costos, traen beneficios sustanciales derivados de la manufactura esbelta. Para ello resulta importante conocer el reordenamiento a las empresas, las que conforme a la Ley 905 de 2004, modificada por la Ley 590 de 2000, según las cuales el sector empresarial está conformado por micro, pequeñas, medianas y grandes empresas. Dicha Ley fue promulgada con el fin de contribuir al fortalecimiento de las mipymes -micro, pequeñas y medianas empresas- a nivel nacional. En este sentido, para el desarrollo de este proyecto, también es importante tener presente, los tipos de empresa como se muestra en la tabla 1. 
Tabla 1. Tipos de empresa

\begin{tabular}{|c|c|c|}
\hline Tipo empresa & Planta de personal & Activos S.M.L.V \\
\hline Pequeña empresa & $11-50$ & $501-5000$ \\
\hline Mediana empresa & $51-200$ & $5001-30000$ \\
\hline
\end{tabular}

Fuente. Elaboración propia con base en la Ley 905 de 2004.

\section{Lean Manufacturing}

\subsection{Enfoque de Lean Manufacturing (LM)}

Es significativo comprender que LM, es un conjunto de prácticas para alcanzar la excelencia y la eliminación de desperdicio, por medio de la mejora continua por el efecto del flujo desde el cliente o sistema Pull; de esta manera LM está basado en una amplia gama de herramientas y técnicas, tales como: Just-in-Time, Gestión Total de la Calidad - TQM, Mantenimiento Productivo Total - TPM, mejoramiento continuo Kaizen, Kanbans, Poka Yoke, Control Estadístico de Procesos, entre otros (Omogbai, 2016).

De otro lado, desde una perspectiva holística se muestra que LM es útil para la fabricación sostenible, lo cual impacta el medioambiente, los aspectos sociales y además la perspectiva económica (Hartini y Ciptomulyono, 2015).

En otros términos, las miradas actuales consideran a LM, como un modelo de gestión que incorpora tanto las herramientas técnicas operativas, como las prácticas de recursos humanos. Según los autores Crjaliu y Draghici (2016), estas herramientas son las siguientes: manufactura celular, Justo a Tiempo (JIT), Kanbans, Mantenimiento Productivo Total (TPM), reducción de tiempos de instalación, Gestión Total de la Calidad (TQM) y 5S. Lo anterior contribuye a la diferenciación de las empresas en un ambiente competitivo, que se fundamenta en los desarrollos desde nuevos diseños innovadores de los productos -artículos con costos óptimos y con atractivos diseños que superen las expectativas del cliente- que los clientes actuales están esperando de los mercados.

\subsection{Casos de estudio Lean y estrategia de operaciones}

Recientemente se ha encontrado que mediante la metodología LM de Toyota y con la aplicación de herramientas Lean se elimina el desperdicio o las demoras, mejora la calidad, la seguridad, la eficiencia y confiabilidad del sistema, al mismo tiempo que disminuye los costos, además de maximizar la eficiencia y la seguridad de las operaciones o en las rutinas de emergencia en el quirófano (Martín, Rampersad, Low y Reed, 2014).

De otro lado, el área metropolitana del Valle de Aburrá agrupó en un estudio a diez sectores económicos, encontrando que la mayor parte de las empresas (85\%) se concentraron en actividades asociadas a la confección, alimentos y bebidas entre otros; y en las que se identificaron trece herramientas duplicadas por ellas, de las cuales aparece el uso Control estadístico de procesos (75\%), Sistema de Gestión ISO 9000 (68\%) y 5S (64\%), entre otras; entre las menos utilizadas: seis sigma, Mantenimiento Productivo Total (TPM) y SMED, con iguales porcentajes (31 \%) (Pérez, Patiño y Úsuga, 2010). 
Otro componente de impacto Lean es el modelo de producción estratégica para devolución de productos de ventas bajo crédito comercial, dado que en los niveles de decisión, la calidad es importante para la gestión de inventarios. Por ello en la gestión del director de operaciones, es determinante que centre su atención en mantener la calidad por lote y determinar la cantidad óptima de pedido para el sistema de inventario, a fin de maximizar el beneficio total esperado por unidad de tiempo (Khanna, Kischore, y Jaggi, 2016).

Por otro lado, es de interés el proceso de implantación de la estrategia en las organizaciones; particularmente se menciona el caso de estudio de una empresa portuaria de Valencia, España, donde el modelo fue estructurado en cuatro partes de la siguiente manera: a) el proyecto de implantación del cuadro de mando integral (C.M.I.); b) la construcción del mapa estratégico; c) la implantación tecnológica y; d) la gestión de la estrategia (Caudeli, Fillol y Ripoll, 2009).

\subsection{Visión y misión empresarial}

Es necesario distinguir en las empresas las tres dimensiones estructurantes del modelo de gestión aplicado: en primer lugar, el simbólico-cultural que trata de generar sentido de identidad incluyente en las compañías, mediante la definición de la visión, misión y los valores corporativos; luego la estratégica y finalmente la dimensión técnico operativo, las tres hacen parte de aspectos fundamentalmente empresariales y de gestión de suma importancia para la alineación total de las empresas (Altuna y Eguzki, 2014).

La misión empresarial tiene por objeto difundir a nivel interno y externo la razón de existencia de la organización, y sirve como guía a los gerentes en la planeación estratégica. Se puede decir que se incluyen en la planeación, de la misión, la imagen pública que se desea, así como los macro objetivos, supervivencia, rentabilidad y crecimiento, todos ellos elementos que deben contribuir a la eficacia de las organizaciones (Klöppel, De Souza y Lunkes, 2015).

Al respecto, en las empresas tecnológicamente avanzadas en Canadá se observaba una correlación importante y alta entre componentes de la visión y misión y el desempeño de las organizaciones, en términos de aplicaciones innovadoras (Robledo y Ríos, 2013).

\subsection{Objetivos de la estrategia de operaciones en calidad y costos, el direccionamiento y decisiones estratégicas}

Como se indicó anteriormente, algunos autores consideran que los componentes de la estrategia de operaciones son: la misión, que es la razón de ser de la organización para la satisfacción de las necesidades de los clientes; la ventaja competitiva, como aspecto diferenciador, objetivos de costo, calidad, flexibilidad y entrega; las decisiones estratégicas con enfoque en el proceso, calidad, capacidad e inventario (Schroeder, Meyer y Rungtusanatham, 2011).

De otra parte, la estrategia de innovación es un factor diferenciador que se soporta en los procesos y la calidad, lo cual parte del direccionamiento estratégico y la formulación de una mega -meta grande y ambiciosa- para el largo plazo, y a un proceso de transformación organizacional, donde hay que declarar la innovación como pilar de la estrategia de negocio, ya que precisa de la calidad (Naranjo y Calderón, 2015).

Una contribución relativa a la disminución de los costos, lo plantea la metodología DMAIC (Definir, Medir, Analizar y Controlar), basada en diferentes herramientas que conllevó al 
logro de la eliminación del desperdicio, en los procesos y operaciones, acortar tiempos de entrega, variabilidad en los procesos e incremento de la productividad (Celis y García, 2013).

Además, unido a lo anterior, va ligado el análisis del peligro, que consiste en la prevención del riesgo, lo que implica la evaluación de la probabilidad que se produzcan este tipo de eventos, y que una vez evaluadas pueden tomarse decisiones en cuanto a cómo enfrentar los riesgos, mediante: eliminación, sustitución, modificación, aislamiento, entre otros. Todos los anteriores contribuyen además a la disminución de costos (Cirjaliu y Draghici, 2016).

Cabe destacar que existe una marcada asociación de las empresas que realizan un proceso integral de direccionamiento estratégico -formulación, implementación y seguimientocon los resultados positivos de la innovación -licenciamiento de patentes y generación de utilidades-, con un estilo proactivo y preactivo de la gestión (Tarapuez, Guzmán y Hernández, 2016).

Así también, para el desarrollo de transformadores culturales, se identifican los formadores de cultura organizacional y se establecen los rasgos culturales ineludibles para la innovación empresarial (Server y Lajara, 2016).

Consecuentemente para lograr la competitividad, la organización debe implementar la planeación estratégica que podrá implementarse de acuerdo con diferentes modelos que conlleva la definición de la estrategia y que cita Porter (2011, p.33), la cual consiste en implementar acciones diferentes, generadas y seleccionadas, que conlleve a las compañías a crear valor. En el mismo sentido, la estrategia proviene de un conjunto de decisiones que se relacionan directamente con el largo plazo de las organizaciones (Uribe y Milena, 2015).

\section{Metodología}

$\mathbf{L}$ a investigación que se desarrolló dentro de este estudio de caso fue mixta, para lo cual se identificaron 7 empresas que fueron el objeto del estudio, seleccionadas mediante muestreo por conveniencia. Fueron ellas: Texmaquila, Sottex, Línea Directa, MIC, Fabricato y otras dos que solicitaron no ser identificadas, dedicadas también a la cadena del sector textil-confección en la ciudad de Medellín, y que implementan técnicas y herramientas que hacen parte de la filosofía Lean. Así mismo, se determinaron aquellas variables que permiten integrar la estrategia operacional con la adopción de herramientas Lean por parte de las compañías del estudio. La muestra se seleccionó a partir del conocimiento en terreno de los investigadores del grado de avance en los procesos de implementación LM en este grupo específico de empresas de la subregión del Valle de Aburrá.

Para la recolección de información se realizó una encuesta estructurada, con preguntas abiertas o generales y un segundo grupo de preguntas cerradas. En el primer tipo de preguntas se indagó por aspectos como nombre de la empresa y del entrevistado, cargo, tiempo de vinculación, número de empleados de planta entre otros; En las preguntas de tipo cerradas, se estableció la escala Likert, con la siguiente valoración: 5 -Muy de acuerdo-, 4 -De 
acuerdo-, 3 -Ni acuerdo o desacuerdo-, 2 -En desacuerdo- y 1 -Muy en desacuerdo-. Estas encuestas se tabularon y luego la información recolectada se analizó y evaluó mediante el apoyo del software Statgraphics Centurion.

La información del estudio se complementó a partir de fuentes secundarias de información como fueron: artículos, informes, tesis, revistas, bases de datos, entre otros, que permitieron la identificación de las variables, factores o aspectos pertinentes a la investigación. Una dificultad o limitante que se presentó fue la dificultad de contactar aquellas empresas del sector textil-confección que hubiesen avanzado significativamente en la implementación Lean, en el Valle de Aburrá Colombia.

\section{Resultados y discusiones}

$\mathrm{E}^{\mathrm{n}}$ la tabla 2 se muestran los resultados en porcentaje de las respuestas a cada una de las preguntas de la encuesta aplicada. Las cifras de la tabla 2 están en porcentajes y en la columna final -total afirmativos- se destaca el acumulado porcentual de las respuestas que se manifiestan de acuerdo o muy de acuerdo, con el componente indicado. Estos porcentajes son el resultado de las respuestas dadas por los encuestados a cada una de las preguntas, las cuales una vez totalizadas, se le establece la ubicación del grupo de respuestas en el nivel de la escala de valoración establecida, la cual como puede observase en las columnas de la tabla 2. Tiene 5 opciones para señalar por parte del encuestado, en cuyos extremos se resalta la opción muy acuerdo para percibir la apreciación de aquel encuestado que está muy identificado con la afirmación hecha en la pregunta y muy en desacuerdo en el caso contrario. 
Tabla 2. Resultados porcentuales en cada una de las preguntas de la encuesta aplicada

\begin{tabular}{|c|c|c|c|c|c|c|}
\hline Ejes De Pregunta & $\begin{array}{l}\text { Muy de } \\
\text { acuerdo }\end{array}$ & $\begin{array}{c}\text { De } \\
\text { acuerdo }\end{array}$ & $\begin{array}{c}\text { En } \\
\text { desacuerdo }\end{array}$ & $\begin{array}{c}\text { Muy en } \\
\text { desacuerdo }\end{array}$ & Indiferente & $\begin{array}{c}\text { Total } \\
\text { afirmativos }\end{array}$ \\
\hline $\begin{array}{l}\text { Incorporación de la filosofía de LM debe } \\
\text { realizarse de forma simultánea en todas } \\
\text { las áreas organizacionales. }\end{array}$ & 42.86 & 42.86 & & 14.29 & - & 85.71 \\
\hline $\begin{array}{l}\text { Se requieren capacitaciones periódicas de } \\
\text { Lean que faciliten la adopción de herra- } \\
\text { mientas de LM. }\end{array}$ & 42.57 & 28.57 & 28.52 & - & - & 71.43 \\
\hline $\begin{array}{l}\text { Incorporar LM para favorecer el mejora- } \\
\text { miento continuo en la organización } \\
\text { favoreciendo la adopción de herramientas } \\
\text { de manufactura esbelta. }\end{array}$ & 85.71 & - & - & 14.29 & - & - \\
\hline $\begin{array}{l}\text { Incorporar LM para el mejoramiento } \\
\text { continuo en la organización favore- } \\
\text { ciendo la adopción de herramientas de } \\
\text { manufactura esbelta. }\end{array}$ & 85.71 & - & - & 14.29 & - & - \\
\hline $\begin{array}{l}\text { Vinculación de la filosofía Lean a la mega y } \\
\text { al direccionamiento estratégico. }\end{array}$ & - & 85.71 & - & 14 & - & - \\
\hline $\begin{array}{l}\text { Estrategia de la innovación, con el conse- } \\
\text { cuente impacto en la implementación de } \\
\text { las estrategias. }\end{array}$ & 42.86 & 28.57 & & 14.29 & 14.29 & 71.43 \\
\hline $\begin{array}{l}\text { La conformación de cooperativas o de los } \\
\text { "clúster» como ventaja competitiva. }\end{array}$ & 42.86 & 14 & - & 14.29 & 14 & 56.86 \\
\hline $\begin{array}{l}\text { Es significativo que la compañía posea } \\
\text { una misión clara y conocida por todos los } \\
\text { empleados de los diferentes niveles jerár- } \\
\text { quicos. }\end{array}$ & 28.58 & 42.86 & - & 14.29 & 28.57 & 71.44 \\
\hline $\begin{array}{l}\text { Es importante la incorporación en las } \\
\text { organizaciones las herramientas LM } \\
\text { ya que le permiten ofertar productos y } \\
\text { servicios únicos al mercado. }\end{array}$ & 28.57 & 28.57 & - & - & 28.57 & 57.14 \\
\hline $\begin{array}{l}\text { La satisfacción de los requerimientos } \\
\text { de los clientes y reducción de costos la } \\
\text { implementación de las herramientas Lean. }\end{array}$ & 57.14 & 14.29 & - & 14.29 & 14.29 & 71.43 \\
\hline $\begin{array}{l}\text { La aplicación de la filosofía Lean de los } \\
\text { productos/servicios ofrecidos por la } \\
\text { compañía. }\end{array}$ & 57.14 & 14.29 & - & 14.29 & 14.29 & 71.43 \\
\hline $\begin{array}{l}\text { La estrategia de innovación, al igual que } \\
\text { las iniciativas y procesos de cambio, se } \\
\text { relacionen con el entorno para acometer } \\
\text { procesos de investigación y desarrollos } \\
\text { soportados en la implementación de los } \\
\text { procesos Lean. }\end{array}$ & 57.14 & - & - & - & 42.86 & - \\
\hline
\end{tabular}


Tabla 2. Resultados porcentuales en cada una de las preguntas de la encuesta aplicada (Continuación)

\begin{tabular}{|l|l|l|l|l|l|l|}
\hline $\begin{array}{l}\text { La visión y misión empresarial este } \\
\text { apropiada por los miembros de la organi- } \\
\text { zación y que se encuentra inmersa la } \\
\text { filosofía Lean para contribuir a los macro } \\
\text { objetivos u objetivos estratégicos. }\end{array}$ & 28.57 & 57.14 & 14.29 & - & - & 85.71 \\
\hline $\begin{array}{l}\text { Reducción en el número de no confor- } \\
\text { midades y la mejora en la calidad de los } \\
\text { productos se encuentren respaldadas en } \\
\text { la implementación estrategias Lean. }\end{array}$ & 42.86 & 42.86 & - & 14.29 & - & 85.71 \\
\hline
\end{tabular}

Fuente. Elaboración propia.

Con base en lo anterior, se puede ver que el $85.71 \%$ de los encuestados poseen una actitud favorable y muy favorable en términos de la necesidad de implementar de manera simultánea las herramientas de Lean Manufacturing en las diferentes áreas organizacionales. Esto implica reducción de costos, estabilización de las curvas de aprendizaje en periodos cortos, cambios unitarios en los modelos de gestión y estructura organizacional. No obstante, la implementación simultánea dependerá de los recursos, capacidades y características de cada organización. Es de resaltar que para el 71.43 $\%$ de los participantes, el éxito en la adopción de LM dependerá en gran medida del número de capacitaciones periódicas para facilitar la interiorización de las herramientas en los colaboradores de la organización.

Por su parte, el $71.44 \%$ de los líderes consultados poseen una actitud muy favorable con respecto al conocimiento que deben poseer todos los colaboradores sobre la misión organizacional. Esto implica el compromiso de los diferentes niveles jerárquicos. A su vez, requiere del diseño claro, preciso y conciso del contenido de la misión, donde se encuentre inmerso el mejoramiento continuo como parte del quehacer diario de los colaboradores. Es así como el $85.71 \%$ de los participantes considera que la incorporación de la filosofía LM en la misión y visión, contribuye con el logro de los objetivos estratégicos.
Resulta relevante señalar que para el $42.86 \%$ de las organizaciones consultadas la estrategia de innovación adelantada en sus operaciones, no se fundamenta en la adopción de herramientas de LM. Dicha estrategia innovadora se centra en componentes externos a la organización, como lo son características y requerimientos del mercado. De aquí que el $28.57 \%$ de los líderes, no posean una actitud muy favorable con respecto a que las herramientas de LM permitan ofertar productos y servicios únicos al mercado.

\subsection{Correlaciones entre variables}

Se analiza el coeficiente de correlación considerando el grado de intensidad o dependencia mutua entre dos variables -para el estudio cada variable se relaciona con una pregunta-, aplicando Statgraphics Centurión de acuerdo con las preguntas contestadas por los encuestados, considerando que si tiende a 1 el grado de correlación o asociación entre las variables será mayor; si tiende a 1 y el $\mathrm{P}$ es $\leq 0,05$, se rechaza el conjunto de hipótesis nula Ho. -ver anexo A-. Además, se identifican en el anexo A, las variables o respuestas de las preguntas dadas a la encuesta, con positiva y fuerte correlación -tienden a 1 y el $\mathrm{P}$ es $\leq 0,05-$.

Como se explica a continuación, en la tabla 3 resumen de correlación de variables, en la cual se muestra el resultado arrojado por el software utilizado, al cruzar las diferentes 
variables y comparar con el coeficiente $\mathrm{P}$, para el cual se estableció un rango igual o menor a 0.05. La tabla presenta en la primera columna la variable a analizar, y en la segunda el resultado de las correlaciones arrojado en la aplicación del respectivo software. En las siguientes columnas se muestran los grados de correlación y el valor de P., en la columna final se establece el significado cualitativo de los valores encontrados.

Tabla 3. Resumen de correlación de variables, con valores hallados al aplicar el software Statgraphics Centurión

\begin{tabular}{|c|c|c|c|c|}
\hline \multicolumn{5}{|c|}{ Resumen correlación de variables } \\
\hline Variable de análisis & Variables correlacionadas & $\begin{array}{l}\text { Grado de } \\
\text { correlación }\end{array}$ & Valor de $\mathrm{P}$ & Análisis \\
\hline \multirow{3}{*}{$\begin{array}{l}\text { Incorporación de } \\
\text { la filosofía de lm } \\
\text { debe realizarse de } \\
\text { forma simultánea } \\
\text { en todas las áreas } \\
\text { organizacionales. }\end{array}$} & $\begin{array}{l}\text { La estrategia de innovación, al igual que } \\
\text { las iniciativas y procesos de cambio, se } \\
\text { relacionen con el entorno para acometer } \\
\text { procesos de investigación y desarrollos } \\
\text { soportados en la implementación de los } \\
\text { procesos lean, con el consecuente impacto } \\
\text { en la implementación de las estrategias. }\end{array}$ & 0.935 & $P \leq 0.02$ & $\begin{array}{c}\text { Fuerte } \\
\text { asociatividad }\end{array}$ \\
\hline & $\begin{array}{l}\text { La visión y misión empresarial esté } \\
\text { apropiada por los miembros de la organi- } \\
\text { zación. }\end{array}$ & 0.944 & $P \leq 0.0014$ & $\begin{array}{c}\text { Altamente } \\
\text { correlacionadas }\end{array}$ \\
\hline & $\begin{array}{l}\text { Es importante la incorporación en las } \\
\text { organizaciones de las herramientas } \mathrm{lm} \text {, } \\
\text { ya que le permiten ofertar productos y } \\
\text { servicios únicos al mercado. }\end{array}$ & 0.8752 & $P \leq 0.099$ & $\begin{array}{l}\text { Positivamente } \\
\text { asociadas }\end{array}$ \\
\hline $\begin{array}{l}\text { Capacitaciones } \\
\text { periódicas de lean que } \\
\text { faciliten la adopción de } \\
\text { herramientas de } 1 \mathrm{~m} .\end{array}$ & $\begin{array}{l}\text { La satisfacción de los requerimientos } \\
\text { de los clientes y reducción de costos la } \\
\text { implementación de las herramientas lean. }\end{array}$ & 0.9526 & $P \leq 0.0009$ & $\begin{array}{l}\text { Están altamente } \\
\text { asociadas }\end{array}$ \\
\hline \multirow{3}{*}{$\begin{array}{l}\text { La incorporación de } \\
\text { lm para favorecer } \\
\text { el mejoramiento } \\
\text { continuo en la } \\
\text { organización. }\end{array}$} & $\begin{array}{l}\text { Incorporar lm para el mejoramiento } \\
\text { continuo en la organización favore- } \\
\text { ciendo la adopción de herramientas de } \\
\text { manufactura esbelta. }\end{array}$ & 0.9685 & $P \leq 0.003$ & $\begin{array}{l}\text { Altamente } \\
\text { asociadas }\end{array}$ \\
\hline & $\begin{array}{l}\text { Vinculación de la filosofía lean a la mega y } \\
\text { al direccionamiento estratégico. }\end{array}$ & 0.9685 & $P \leq 0.0003$ & $\begin{array}{c}\text { Altamente } \\
\text { correlacionados }\end{array}$ \\
\hline & $\begin{array}{l}\text { Reducción en el número de no } \\
\text { conformidades y la mejora en la calidad de } \\
\text { los productos se encuentren respaldadas } \\
\text { en la implementación estrategias lean. }\end{array}$ & 0.9366 & $P \leq 0.019$ & $\begin{array}{l}\text { Altamente } \\
\text { asociadas }\end{array}$ \\
\hline
\end{tabular}


Tabla 3. Resumen de correlación de variables, con valores hallados al aplicar el software Statgraphics Centurión (Continuación)

\begin{tabular}{|c|c|c|c|c|}
\hline \multicolumn{5}{|c|}{ Resumen correlación de variables } \\
\hline Variable de análisis & Variables correlacionadas & $\begin{array}{l}\text { Grado de } \\
\text { correlación }\end{array}$ & Valor de $\mathrm{P}$ & Análisis \\
\hline \multirow{4}{*}{$\begin{array}{c}\text { Vinculación de la } \\
\text { filosofía lean a la mega } \\
\text { y al direccionamiento } \\
\text { estratégico. }\end{array}$} & $\begin{array}{l}\text { La visión y misión empresarial esté } \\
\text { apropiada por los miembros de la organi- } \\
\text { zación. }\end{array}$ & 0.9058 & $P \leq 0.005$ & $\begin{array}{l}\text { Positivamente } \\
\text { asociadas }\end{array}$ \\
\hline & $\begin{array}{l}\text { La satisfacción de los requerimientos } \\
\text { de los clientes y reducción de costos la } \\
\text { implementación de las herramientas lean. }\end{array}$ & 0.8023 & $P \leq 0.0299$ & $\begin{array}{l}\text { Positivamente } \\
\text { asociadas }\end{array}$ \\
\hline & $\begin{array}{l}\text { Incorporar lm para el mejoramiento } \\
\text { continuo en la organización favore- } \\
\text { ciendo la adopción de herramientas de } \\
\text { manufactura esbelta. }\end{array}$ & 0,8023 & $P \leq 0.0299$ & $\begin{array}{l}\text { Son variables } \\
\text { positivamente } \\
\text { asociadas }\end{array}$ \\
\hline & $\begin{array}{l}\text { Es significativo que la compañía posea } \\
\text { una misión clara y conocida por todos } \\
\text { los empleados de los diferentes niveles } \\
\text { jerárquicos. Apropiada por los miembros } \\
\text { de la organización. }\end{array}$ & 0.9806 & $P \leq 0.0001$ & $\begin{array}{c}\text { Variables } \\
\text { altamente } \\
\text { correlacionadas }\end{array}$ \\
\hline $\begin{array}{l}\text { Estrategia de la } \\
\text { innovación, con el } \\
\text { consecuente impacto } \\
\text { en la implementación } \\
\text { de las estrategias. } \\
\end{array}$ & $\begin{array}{l}\text { Reducción en el número de no confor- } \\
\text { midades y la mejora en la calidad de los } \\
\text { productos se encuentren respaldadas en } \\
\text { la implementación estrategias lean. }\end{array}$ & 0.8922 & $P \leq 0.0069$ & $\begin{array}{l}\text { Variables con } \\
\text { alta correlación }\end{array}$ \\
\hline $\begin{array}{l}\text { Es importante la } \\
\text { incorporación en las } \\
\text { organizaciones de las } \\
\text { herramientas lm ya } \\
\text { que le permiten ofertar } \\
\text { productos y servicios } \\
\text { únicos al mercado. }\end{array}$ & Mejora en la calidad de los productos. & 0.8922 & $P<=0.0069$ & $\begin{array}{l}\text { Son variables } \\
\text { con alta } \\
\text { correlación }\end{array}$ \\
\hline \multirow{3}{*}{$\begin{array}{l}\text { Incorporar lm para } \\
\text { el mejoramiento } \\
\text { continuo en la } \\
\text { organización } \\
\text { favoreciendo } \\
\text { la adopción de } \\
\text { herramientas de } \\
\text { manufactura esbelta. }\end{array}$} & $\begin{array}{l}\text { La satisfacción de los requerimientos } \\
\text { de los clientes y reducción de costos la } \\
\text { implementación de las herramientas lean. }\end{array}$ & 0.8922 & $P<=0.0069$ & $\begin{array}{l}\text { Variables } \\
\text { con positiva } \\
\text { asociatividad }\end{array}$ \\
\hline & $\begin{array}{l}\text { Vinculación de la filosofía lean a la mega y } \\
\text { al direccionamiento estratégico. }\end{array}$ & 0.9255 & $\mathrm{P}<=0.028$ & $\begin{array}{l}\text { Se evidencia } \\
\text { alta correlación }\end{array}$ \\
\hline & $\begin{array}{l}\text { Reducción en el número de no confor- } \\
\text { midades y la mejora en la calidad de los } \\
\text { productos se encuentren respaldadas en } \\
\text { la implementación estrategias lean. }\end{array}$ & 0.8922 & $P \leq 0.0069$ & $\begin{array}{l}\text { Son variables } \\
\text { con positiva } \\
\text { asociatividad }\end{array}$ \\
\hline
\end{tabular}

Fuente. Elaboración propia. 
Se aprecia en los resultados que la mayoría de variables de análisis presentan un alto o fuerte grado de asociatividad o correlación términos que para el presente estudio deben tomarse como sinónimos-, sin embargo, dos resultados de la tabla tienen un grado de correlación levemente inferior al promedio anterior -valor de la correlación mayor a 0.80 con $\mathrm{P}<0.05-$, por lo que en la valoración hecha en el análisis se catalogaron como positivamente asociadas.

De esta manera, la incorporación simultánea del LM en las áreas organizacionales posee una alta correlación con el nivel de apropiación que posean los colaboradores con la misión y visión; además de poseer una fuerte asociatividad con la estrategia de innovación. Esto implica la coordinación entre los lineamientos estratégicos y la interiorización de los requerimientos del entorno. En consecuencia, las capacitaciones en adopción LM expresan un alto nivel de asociatividad con la satisfacción de las necesidades de los consumidores y la reducción de los costos de implementación.

Por otro parte, la vinculación de LM en la mega y direccionamiento estratégico manifiesta un alto nivel de correlación con el mejoramiento continuo de la organización. Lo anterior se encuentra reflejado en el alto nivel de asociatividad que obtuvo esta variable, con la interiorización del LM por parte de todos los colaboradores, independientemente de su ubicación en los diferentes niveles jerárquicos. En efecto, el LM requiere del compromiso y apropiación por parte de la alta dirección, además del ejemplo como la muestra del convencimiento que estos manifiesten a los demás colaboradores.
Ahora bien, la calidad se destaca como uno de los objetivos estratégicos más relevantes en la incorporación de LM. Es así como esta última posee una correlación significativa en la mejora en la calidad de los productos y servicios ofertados, lo que repercute en la satisfacción eficiente de las necesidades del consumidor. Además, la calidad también se refleja en los procesos internos, de forma especial en la reducción de mudas, desperdicios y no conformidades (tabla 3 ).

Se desprende del análisis que, al incorporar LM para el mejoramiento continuo en la organización, favorece la adopción de herramientas de manufactura esbelta, además de que con la vinculación de la filosofía Lean a la mega y al direccionamiento estratégico (Altuna y Eguzki, 2014), se impacta la reducción del número de no conformidades y consecuentemente en la mejora de la calidad de los productos ofrecidos a los clientes, lo que permite ofertar productos y servicios competitivos en el mercado. Así también, la vinculación de la filosofía Lean a la mega y al direccionamiento estratégico (Altuna y Eguzki, 2014), facilita la incorporación LM para el mejoramiento continuo en la organización potenciando la adopción de herramientas de manufactura esbelta.

De otra parte, las capacitaciones periódicas de Lean Manufacturing que facilitan la adopción en la empresa de estas herramientas manufactura celular, Justo a Tiempo (JIT)-, Kanbans, Mantenimiento Productivo Total (TPM), reducción de tiempos de instalación, Gestión Total de la Calidad (TQM) y 5S (Cirjaliu y Draghici, 2016), permiten evidenciar la satisfacción de los requerimientos de los clientes y la reducción de costos mediante la implementación de las herramientas Lean. 


\subsection{Propuestas teóricas: estrategias de operaciones: calidad y costos}

A continuación, se presenta un resumen de las dos propuestas teóricas que hacen parte de los productos finales de este estudio de caso.

\subsubsection{Propuesta teórica 1.}

En la figura 1 se observan la interrelaciones entre las estrategias y la filosofía Lean, con aspectos claves identificados como resultado de la investigación realizada en todos los componentes del modelo Lean: misión clara y conocida por los empleados, incorporación Lean a todas las áreas, LM como mejora de la calidad y reducción de las no conformidades; la misión, la filosofía Lean y los macro objetivos; la satisfacción de los clientes y la reducción de costos, uso de los recursos tecnológicos, favorecer el mejoramiento continuo de las empresas, aplicación de las herramientas Lean, vinculación de la filosofía Lean y la estrategia de la innovación.

Figura 1. Estrategias y filosofía Lean

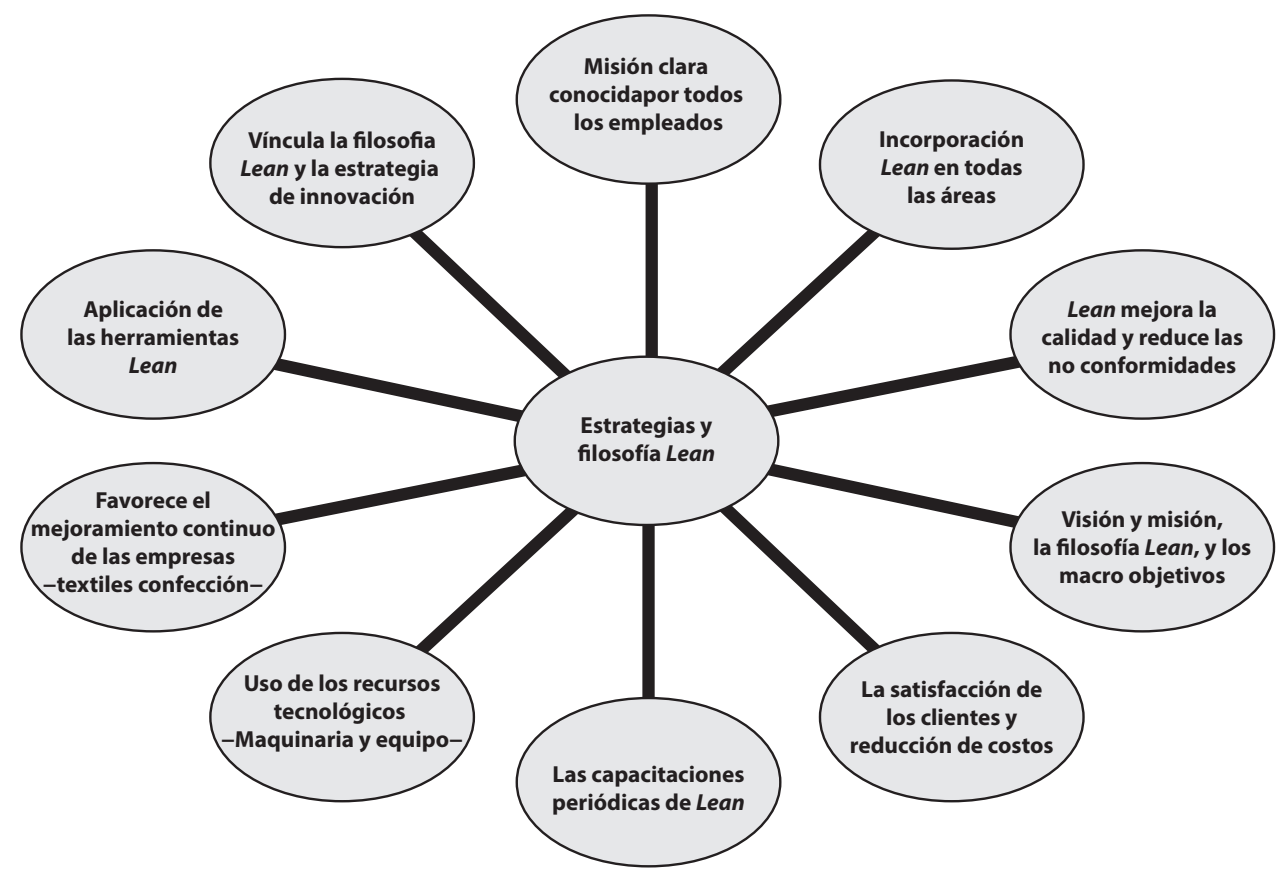

Fuente. Elaboración propia.

Las estrategias de calidad y costos, y la filosofía Lean se articulan según la figura 1, como sigue:

- Visión y misión y los macro objetivos: en la filosofía organizacional se encuentra inmersa la visión y misión, y con el propósito de desarrollar el direccionamiento se definen los objetivos estratégicos: supervivencia, rentabilidad y crecimiento, a su vez las estrategias de largo plazo, en la que deben definirse además las estrategias Lean y la filosofía Lean (Klöppel et al., 2015).

- Misión clara y conocida por todos los niveles jerárquicos: la visión se cumple con la misión, la cual debe ser difundida 
en todos los niveles de la compañía, para que todos se empoderen de ella (Robledo y Ríos, 2013).

- Incorporación Lean en todas las áreas: para desarrollar una ventaja competitiva de la compañía es indispensable incorporar Lean en todas las áreas de la empresa (Robledo y Ríos, 2013).

- Lean mejora de la calidad y reducción de no conformidades: al incorporar Lean en toda la compañía mejora la calidad y reduce las no conformidades. (Pérez et al., 2010).

- La satisfacción de clientes y reducción de costos: Lean en toda la organización, logra la satisfacción de los requerimientos de los clientes y consecuentemente reduce los costos (Celis y García, 2013).

- Las capacitaciones periódicas de Lean: es indispensable además realizar capacitaciones periódicas a todo el personal. (Uribe y Milena, 2015).

- Uso de recursos tecnológicos: en consecuencia, para el desarrollo de Lean la compañía deberá contar con tecnología de punta en maquinaria y equipo (Caudeli et al., 2009).

- Favorecen el mejoramiento continuo de las empresas: también la estrategia de operaciones y la filosofía Lean favorece el mejoramiento continuo de las organizaciones (Alukal y Manos, 2006).
- Aplicación de las herramientas Lean: para un óptimo desarrollo de la filosofía Lean y la estrategia de operaciones es indispensable la aplicación de las herramientas Lean, entre las cuales se puede citar: manufactura celular, Justo a Tiempo (JIT), Kanbans, reducción de tiempos de instalación, $5 \mathrm{~S}$, entre otros (Cirjaliu y Draghici, 2016).

- Vincular la filosofía Lean a la estrategia de la organización: la estrategia de la organización es de largo plazo, razón por el cual resulta indispensable vincular la filosofía como una estrategia de la organización (Uribe y Milena, 2015).

- Filosofía Lean y la estrategia de la innovación: está estrechamente vinculada, ya que la propuesta de valor está contenida en los productos o servicios que son ofrecidos a los clientes (Naranjo y Calderón, 2015).

\subsubsection{Propuesta teórica 2.}

Se elaboró la siguiente propuesta teórica, en la que se visualiza gráficamente cada uno de los procesos que deberá seguir la compañía que busque ser exitosa, en la interrelación de estrategias de operaciones de calidad y costos, con la incorporación de la filosofía Lean, y las decisiones estratégicas. Se logran identificar en la figura 2. los procesos de Direccionamiento Estratégico, Innovación y Capacitación Lean, en todas las áreas: calidad, reducción de no conformidades y costos, servicio al cliente. 
Figura 2. Estrategias de operaciones: calidad y costos e incorporación de la filosofía Lean

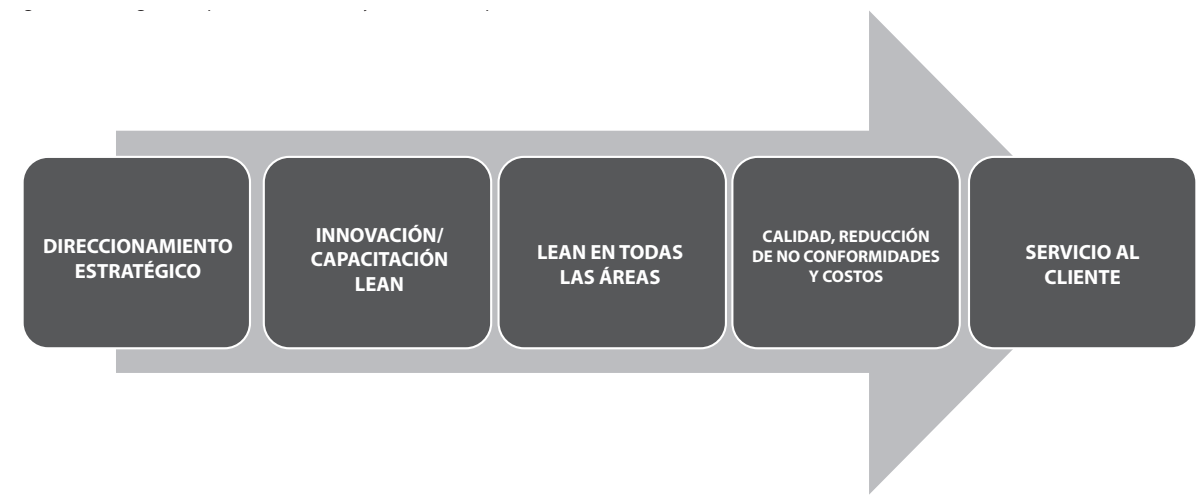

Fuente. Elaboración propia.

Para la incorporación de la filosofía Lean y la estrategia de operaciones de calidad y costos a la compañía, es indispensable que se distingan los procesos de direccionamiento estratégico de la empresa, igualmente definir y difundir a todos los niveles de la organización la mega, la visión, visión y valores, además se definan los objetivos estratégicos y estrategias de largo plazo, entre los cuales deberá incorporarse la innovación como uno de ejes estratégicos claves (Naranjo y Calderón, 2015).
Lo anterior deberá estar acompañado de la capacitación Lean en todos los niveles de la organización, buscando generar empoderamiento del personal e incorporación de Lean en todas las áreas, uso de las herramientas Lean, incorporación de tecnología, lo cual se verá reflejado tanto en la calidad final del producto, así como la reducción de las no conformidades y costos por parte del cliente. Finalmente, lo anterior coadyuva al mejoramiento continuo y al cumplimiento de los requerimientos de los clientes y la lealtad de estos en el largo plazo. (Celis \& García, 2013).

\section{Conclusiones}

L as particularidades más relevantes del estudio que soporta el presente artículo, encuentra que las compañías como Mic y Sottex abordan la estrategia de calidad con la consecuente mejora de no conformidades, empoderando la misión a todos los empleados, alineando de forma total los objetivos estratégicos a la visión, aunado a la estrategia de innovación. Así mismo, Línea Directa y Fabricato, desarrollan las estrategias de calidad y costos, aplican algunas herramientas Lean, lo cual favorece el mejoramiento continuo, mediante el uso de tecnología de punta. También la empresa Texmaquila desarrolla la estrategia de costos y aplica algunas herramientas Lean -ver variables en la figura 1-, además se encontraron correlaciones altas entre la vinculación de la mega al direccionamiento estratégico con la apropiación de la misión en todos los niveles jerárquicos de las empresas, pertenecientes al sector textil-confección en el Valle de Aburrá (Tabla 2). 
También, se logró identificar que mediante la integración Lean en todas las áreas de la organización empresarial, se desarrolla una ventaja competitiva en las compañías, y con la adopción de las dos propuestas (Figura 1 y 2) de orden administrativo, descritas en el artículo, las cuales están basadas en la estrategia de operaciones, calidad y costos, permiten la optimización en la implementación de estas herramientas. Se concluye, además, que este aspecto aunado al enfoque de la filosofía empresarial identificado en la propuesta de la figura 1 -la mega, la visión, la misión de la compañía y los objetivos estratégicos- si es empoderado por todos los niveles jerárquicos de la empresa, conlleva a que los integrantes de las organizaciones del sector textil confección trabajen en el día a día en una misma dirección, maximizando la productividad e incrementando la rentabilidad de las empresas.

Igualmente, entre los principales factores de la implementación de herramientas de Lean Manufacturing antes citadas, se identificaron el direccionamiento estratégico, la estrategia de innovación, la calidad y costos entre otros -propuesta de la figura 2-, lo que coadyuva a que el responsable de la gerencia de la compañía, si aplica las propuestas teóricas sugeridas por el estudio, podrá cumplir los requerimientos de los clientes, aspecto que impacta el cumplimiento de la promesa del servicio o del producto entregado a los clientes y a la vez contribuye a la lealtad de estos en el largo plazo, así como a la permanencia de las empresas del sector investigado en un mercado cada vez más competitivo.

El empoderamiento del direccionamiento estratégico de la empresa en todos los niveles jerárquicos, es un elemento crítico en la implementación de las propuestas -figura 1por lo tanto las capacitaciones periódicas de los actores involucrados son un elemento clave para lograr niveles eficientes de compromiso y pertenencia, con lo que se demuestra que Lean contribuye a la mejora de la calidad y reduce las no conformidades, satisface los clientes y reduce costos, haciendo uso óptimo de los recursos -maquinaria y equipo-, favorece el mejoramiento continuo de las empresas del sector textil-confección entre otros, luego se puede decir que la manufactura esbelta puede considerarse una estrategia para mitigar algunas de las ineficiencias en el uso de recursos, corroborando los planteamientos de algunos teóricos expertos en el tema. Este aspecto unido con el uso en el sector de las empresas textil confección de recursos de tecnología de punta, maquinaria y equipo, con el desarrollo Lean entre otros, posibilitará que las empresas permanezcan en el mercado y consigan crecer en el mediano y largo plazo.

En consecuencia, con los hallazgos y análisis del estudio presentado en este artículo, se considera importante la identificación de los elementos que contribuyen a que las compañías dedicadas a la confección en la ciudad de Medellín y Valle de Aburrá, implementen las herramientas LM -Kanban, 5S, Kaizen, Just-inTime, Gestión Total de la Calidad - TQM, entre otras- para que favorezcan el mejoramiento continuo, fortalezcan la competitividad en el mercado y logren garantizar la permanencia de sus clientes. En general, la ejecución de la estrategia de operaciones de calidad y costos, aunado al compromiso de la filosofía Lean en toda la organización, maximiza el beneficio esperado por unidad de tiempo en todos los procesos, propiciando un uso óptimo de los recursos, con los consabidos beneficios en términos del cumplimiento de la misión empresarial. La aplicación de técnicas, herramientas y filosofía Lean, que es escasa en algunas empresas del sector textil-confección, se corroboró en el estudio realizado para las empresas pequeñas y medianas de dicho sector ubicadas en la subregión del Valle de Aburrá y participes del estudio. 


\section{Referencias}

Altuna, R. (2014). La cooperativa Fagor Ederlan: el Lean Manufacturing como modelo de gestión. España: CIRIEC.

Alukal, G. Manos, A. (2006). A Simplified Approach To Process Improvements. En Lean Kaizen. Milwaukee: A Hytinen \& P O'Mara Editores.

Arrieta, J.. (2010). Benchmarking sobre manufactura esbelta (Lean Manufacturing) en el sector de la confección en la ciudad de Medellín. Colombia Journal of Economics, Finance and Administrative Science.

Caudeli, J. A., Fillol, A. G., Feliú, V., y Ripoll, M. (2009). Análisis del proceso de implantación de un sistema de gestión estratégica: estudio de caso cuadro de mando integral en la autoridad portuaria de Valencia. http:// dx.doi.org/10.1080/02102412.2009.10779666

Celis, O. L. y García J.M. (2013). Modelo tecnológico para el desarrollo de proyectos logísticos usando Lean Six Sigma. Estudios Gerenciales, 28(124), 23-43.

CEPAL. (2010). Politicas de apoyo a las pyme en America Latina, entre avances innovadores y desafios institucionales, 13.

Cirjaliu, B. y Draghici, A. (2016). Ergonomic Issues in Lean Manufacturing. Procedia - Social and Behavioral Sciences, 221, 105-110. https://doi. org/10.1016/j.sbspro.2016.05.095

Hartinia, S. y Ciptomulyonob, U. (2015). The Relationship between Lean and Sustainable Manufacturing on Performance: Literature Review. Procedia Manufacturing, 4, 38-45. https://doi. org/10.1016/j.promfg.2015.11.012

Khanna, K. (2016). Strategic production modeling for detective with imperfect inspetion process, rework, and sales return under two-level trade credit2016. In G. Science (Ed.) International Journal of Industrial Engineering Computations , 8(30), 85-118.

Klöppel, F., de Souza, P., y Lunkes, R. J. (2015). Missao empresarial: uma análise de sua efectividade para o planejamento estratégico. Contaduría Universidad de Antioquia, 66, 113-129.

Ley 905. (2004). Ley mipyme. Bogota, Colombia: Congreso de la República.
Madariaga, F. (2013). Lean Manufacturing. Bubok PublishingS.L. Recuperado de https://books.google. $\mathrm{com} /$ books? id=mBgDGYRQzXMC\&pgis $=1$

Martín, L. D., Rampersad, S. E., Low, D. K., y Reed, M. A. (2014). Mejoramiento de los procesos en el quirófano mediante la aplicación de la metodología Lean de Toyota. Revista Colombiana de Anestesiologia , 42(3), 220-228.

Naranjo, J., y Calderón, G. (2015, abril-junio). Construyendo una cultura de innovación. Una propuesta de transformación cultural. Estudios Gerenciales, 31(135), 223-236. Recuperado de http://www.sciencedirect. com/science/article/pii/S0123592315000054

Omogbai, K. S. (2016). Variation modeling of Lean Manufacturing performance using fuzzy logic. Recuperado de http://www.sciencedirect.com/ science/article/pii/S2212827116000196

Pérez, R., Patiño, R., y Úsuga, M. (2010). Uso de herramientas de mejoramiento y su incidencia en costos, fallas y factores de éxito de grandes y medianas empresas industriales del Valle de Aburrá . GyP, 589-602. Recuperado de http://dx.doi. org/10.1590/S0104-530X2010000300012

Porter, M. E. (2011). The Competitive Advantage Of Nations. New York: Freee Press.

Rajadel, M. y Sánchez, J. L. (2010) Lean Manufacturing, evidencia de una necesidad. España: Ediciones Díaz de Santos. Recuperado de http://books.google.com.co/ books/about/Lean_Manufacturing_La_evidencia_ de_una_n.html? id= $=1$ R2xgsdmdUoC\&pgis $=1$

Robledo C. y Ríos C. (2013). La internacionalización como objetivo explícito en la visión y misión declaradas de 500 empresas más grandes en Colombia. AD-minister, (22), 33-50. Recuperado de http://www.redalyc.org/ pdf/3223/322327380003.pdf

Schroeder, R., Meyer, S., y Rungtusanatham, M. J. (2011). Administración de operaciones: conceptos y casos contemporáneos (5.a ed.). México. Recuperado de http://www.casadellibro.com/libroadministracion-de-operaciones-conceptos-y-casoscontemporaneos--5-ed/9786071506009/1863736 
Tarapuez, E., Guzmán, B., y Hernández, R. (2016). Estrategia e innovación en las mipymes colombianas ganadoras del premio Innova 20102013. Estudios Gerenciales, 32(139). Recuperado de http://www.sciencedirect.com/science/article/pii/ S012359231630002X

Server, R. J., y Lajara, N. (2013). Factores coadyuvantes delainnovación en las cooperativas agroalimentarias. Caso estudio de las citrícolas españolas. Revista Itea Información Técnica y Económica Agraria, 112(2), 185199. Recuperado de http://www.aida-itea.org/aidaitea/files/itea/revistas/2016/112-2/ITEA\%201122.pdf\#page $=81$

Uribe, M. E., y Milena, J. C. (2015). Experiencia y resultados de la aplicación de la técnica de consenso de panel Uso empírico para la selección de dos organizaciones tolimenses. Revista ClioAmérica, 9(18). Recuperado de http://revistas.unimagdalena. edu.co/index.php/clioamerica/article/view/1530

Vienazindiene, M., y Ciarniene, R. (2013). Lean Manufacturing Implementation and Progress Measurement. Economics and Management, 18(2). http://dx.doi.org/10.5755/j01.em.18.2.4732 


\section{Anexo A}

Correlación entre variables o variables bivariadas.

\begin{tabular}{|c|c|c|c|c|c|c|c|c|c|c|c|c|c|c|}
\hline & PG1 & PG2 & PG3 & PG4 & PG5 & PG6 & PG7 & PG8 & PG9 & PG10 & PG11 & PG12 & PG13 & PG14 \\
\hline \multirow[t]{3}{*}{ PG1 } & & 0,4617 & 0,5914 & 0,5509 & 0,747 & 0,5535 & 0,5603 & 0,5762 & 0,2395 & 0,3841 & 0,3841 & 0,1956 & 0,565 & 0,3843 \\
\hline & & 7 & 7 & 7 & 7 & 7 & 7 & 7 & 7 & 7 & 7 & 7 & 7 & 7 \\
\hline & & 0,297 & 0,162 & 0,1999 & 0,0536 & 0,1974 & 0,1908 & 0,1758 & 0,6049 & 0,3949 & 0,3949 & 0,6743 & 0,1863 & 0,3947 \\
\hline \multirow[t]{3}{*}{ PG2 } & & & 0,7464 & 0,665 & 0,7344 & 0,4767 & 0,5856 & 0,4763 & 0,2546 & 0,9526 & 0,8845 & 0,6236 & 0,5204 & 0,7506 \\
\hline & & & 7 & 7 & 7 & 7 & 7 & 7 & 7 & 7 & 7 & 7 & 7 & 7 \\
\hline & & & 0,054 & 0,1031 & 0,0601 & 0,2794 & 0,1672 & 0,2799 & 0,5817 & 0,0009 & 0,0082 & 0,1345 & 0,2311 & 0,0519 \\
\hline \multirow[t]{3}{*}{ PG3 } & & & & 0,9685 & 0,9685 & 0,8607 & 0,8 & 0,866 & 0 & 0,866 & 0,866 & 0,441 & 0,9058 & 0,9366 \\
\hline & & & & 7 & 7 & 7 & 7 & 7 & 7 & 7 & 7 & 7 & 7 & 7 \\
\hline & & & & 0,0003 & 0,0003 & 0,0129 & 0,0307 & 0,0117 & 1 & 0,0117 & 0,0117 & 0,322 & 0,005 & 0,0019 \\
\hline \multirow[t]{3}{*}{ PG4 } & & & & & 0,9255 & 0,935 & 0,7128 & 0,8752 & $-0,136$ & 0,8023 & 0,8023 & 0,3342 & 0,944 & 0,8518 \\
\hline & & & & & 7 & 7 & 7 & 7 & 7 & 7 & 7 & 7 & 7 & 7 \\
\hline & & & & & 0,0028 & 0,002 & 0,0722 & 0,0099 & 0,7705 & 0,0299 & 0,0299 & 0,4638 & 0,0014 & 0,015 \\
\hline \multirow[t]{3}{*}{ PG5 } & & & & & & 0,8589 & 0,8617 & 0,8023 & 0 & 0,8023 & 0,8023 & 0,3342 & 0,8439 & 0,8518 \\
\hline & & & & & & 7 & 7 & 7 & 7 & 7 & 7 & 7 & 7 & 7 \\
\hline & & & & & & 0,0133 & 0,0127 & 0,0299 & 1 & 0,0299 & 0,0299 & 0,4638 & 0,017 & 0,015 \\
\hline \multirow[t]{3}{*}{ PG6 } & & & & & & & 0,6632 & 0,7454 & $-0,279$ & 0,5963 & 0,6708 & 0 & 0,8478 & 0,665 \\
\hline & & & & & & & 7 & 7 & 7 & 7 & 7 & 7 & 7 & 7 \\
\hline & & & & & & & 0,1044 & 0,0545 & 0,5447 & 0,1576 & 0,099 & 1 & 0,016 & 0,1031 \\
\hline \multirow[t]{3}{*}{ PG7 } & & & & & & & & 0,5105 & $-0,273$ & 0,6564 & 0,5835 & 0,1114 & 0,5578 & 0,7217 \\
\hline & & & & & & & & 7 & 7 & 7 & 7 & 7 & 7 & 7 \\
\hline & & & & & & & & 0,2417 & 0,5538 & 0,1093 & 0,1691 & 0,812 & 0,1932 & 0,0671 \\
\hline \multirow[t]{3}{*}{ PG8 } & & & & & & & & & 0,1336 & 0,6429 & 0,6429 & 0,5455 & 0,9806 & 0,8111 \\
\hline & & & & & & & & & 7 & 7 & 7 & 7 & 7 & 7 \\
\hline & & & & & & & & & 0,7752 & 0,1194 & 0,1194 & 0,2053 & 0,0001 & 0,0268 \\
\hline \multirow[t]{3}{*}{ PG9 } & & & & & & & & & & 0,1336 & 0,2673 & 0,6124 & 0 & 0,1517 \\
\hline & & & & & & & & & & 7 & 7 & 7 & 7 & 7 \\
\hline & & & & & & & & & & 0,7752 & 0,5623 & 0,1438 & 1 & 0,7453 \\
\hline \multirow[t]{3}{*}{ PG10 } & & & & & & & & & & & 0,9286 & 0,6547 & 0,6864 & 0,8922 \\
\hline & & & & & & & & & & & 7 & 7 & 7 & 7 \\
\hline & & & & & & & & & & & 0,0025 & 0,1106 & 0,0886 & 0,0069 \\
\hline \multirow[t]{3}{*}{ PG11 } & & & & & & & & & & & & 0,5455 & 0,6864 & 0,8922 \\
\hline & & & & & & & & & & & & 7 & 7 & 7 \\
\hline & & & & & & & & & & & & 0,2053 & 0,0886 & 0,0069 \\
\hline \multirow[t]{3}{*}{ PG12 } & & & & & & & & & & & & & 0,4494 & 0,6195 \\
\hline & & & & & & & & & & & & & 7 & 7 \\
\hline & & & & & & & & & & & & & 0,3118 & 0,1379 \\
\hline \multirow[t]{3}{*}{ PG13 } & & & & & & & & & & & & & & 0,8113 \\
\hline & & & & & & & & & & & & & & 7 \\
\hline & & & & & & & & & & & & & & 0,0268 \\
\hline \multicolumn{15}{|l|}{ PG14 } \\
\hline & & & & & & & & & & & & & & \\
\hline
\end{tabular}

Fuente. Elaboración propia. 